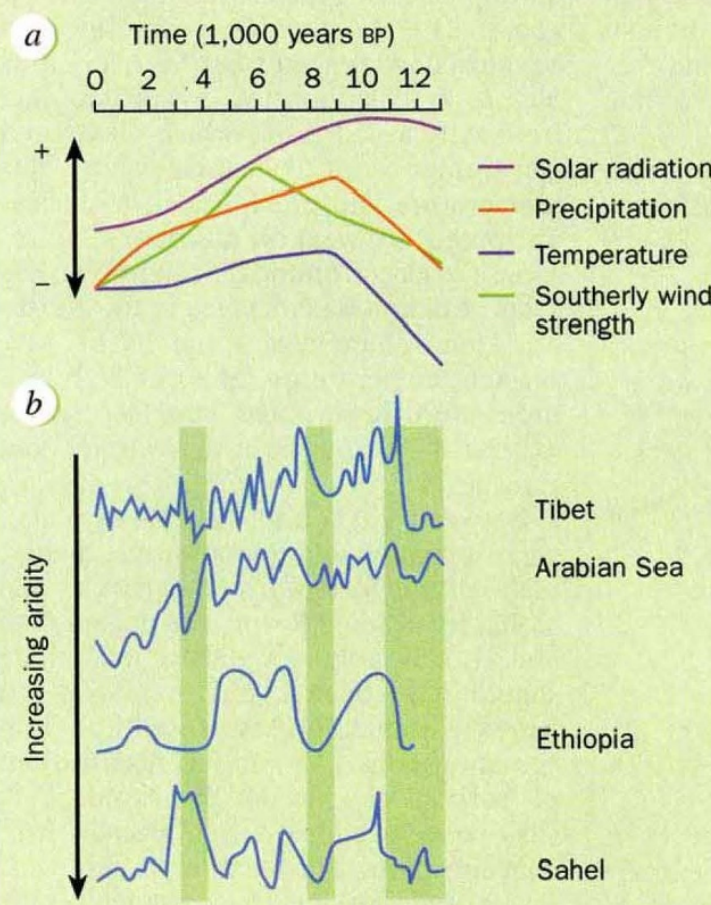

Climate change and droughts throughout tropicalsubtropical Asia and Africa during the past 13,000 years. a, The variation of solar insolation ${ }^{11}$ and environmental para meters; temperature, precipitation and southerly wind strength are inferred from a numerical climate model ${ }^{6} . b$, Four different environmental records, each indicating relative aridity: for Tibet, a pollen record from Lake Sumxi in the western Tibetan highlands, indicating changes in vegetation ${ }^{4}$; Arabian Sea, variations in carbonate concentration along a sediment core drilled off the coast of Oman (decreasing carbonate concentrations after 5,500 years BP indicate increased deposition of airborne dust because of enhanced aridity in Arabia $^{12}$ ); Ethiopia, water level variation in Lake Abyata in the Ethiopian rift $^{4}$; Sahel, an oxygen isotope record of a former lake in the Chad basin ${ }^{4}$ (variations in isotope composition of the ancient lake's water were driven by the balance between precipitation and evaporation). In each case, aridity increases downwards. Major dry spells occurred at $11,000-13,000,8,000-9,000$ and $3,000-4,000$ years ago (all ages are given here in calendar years). The data also show differences in climate trend as one moves from the Indian monsoon domain to that of Atlantic Africa. Highlatitude forcing of climates in Atlantic Africa may account for the differences; changes in heat transport to the north in the Atlantic sector of the global thermohaline 'conveyor-belt' presumably affects the world-wide pattern of interhemispheric temperature contrast which determines the moisture flux to tropical Africa ${ }^{10}$.

past 80 years, dry episodes including the current drought in the Sahel have been correlated with warming of the surface ocean in the Southern Hemisphere and the North Indian Ocean, and cooling of the North Atlantic and the North Pacific $^{10}$. The net effect of this world-wide SST anomaly pattern was a reduction of moisture flux into the Sahel zone. Such changes may help to explain the climate pattern of a restricted geographical region, although they may not uniquely explain the pattern of Holocene climate throughout tropical and subtropical Africa and Asia.

Two steps seem to be essential if we are to gain better knowledge about coupled ocean and atmospheric forcing of continental climate at all latitudes. First, atmospheric general circulation models ought to be coupled to a dynamic ocean thermohaline circulation which changes in accord with changing external boundary conditions (and ideally also with changing internal boundary conditions which follow from the external changes). Models do not properly account for this at present; the NASA GISS model, for instance, which was used to test monsoonal sensitivity to external boundary conditions ${ }^{7}$, prescribes ocean heat fluxes in a series of 'correction fluxes' which vary as a function of latitude so as to balance the offset between purely insolation-driven and observed atmospheric temperature gradients. That is, the real ocean's dynamics are excluded from the sensitivity test, thus limiting the output to the atmosphere's dynamics.

Second, hard palaeo-data are needed to test and refine the models. Ideally, the data would be acquired from marine sediment cores taken along the flow path of the upper limb of the ocean's conveyor belt. Critical areas to be studied are the Pacific-Indian heatflow pathway through the Indonesian archipelago, the Algulhas current and associated flow of surface water and heat around South Africa into the South Atlantic, the North Atlantic and North Pacific convection and return-flow regimes, and the complex equatorial current systems. Research programmes such as MESH (Marine Aspects of Earth System History) and IMAGES (International Marine Global Change Study) are just appearing on the horizon of global change studies to guide palaeo-oceanography and palaeoclimate research into the next century. As such, they should provide the international and multidisciplinary forum needed to carry out such research.

Rainer Zahn is at the GEOMAR Research Centre for Marine Geosciences, Wischhofstrasse 1-3, $24148 \mathrm{Kiel}$, Germany.

\section{Scan the Heavens}

Now that the heroic succession of interplanetary space probes is beginning to falter, Daedalus is thinking of other ways to study our planetary neighbours. He points out that, like the solar wind, a particle beam could travel through space almost indefinitely. Electron beams can be generated with peak energies of many gigawatts; during the Star Wars project they were seriously considered as space weapons. So Daedalus is inventing the scanning electron telescope.

His idea is to put a beam-launching satellite into some low orbit, and aim intense particle beams at the planets. The obvious problem is chargeneutrality. A satellite which launched a single strong electron beam would accumulate such a vast positive charge that it would soon attract the beam disastrously back onto itself. It would have to launch an electron beam and a positive-ion beam at the same time, and ideally at the same target. The two parallel beams would then attract each other. In the course of their journey, they might entwine to form, in effect, a stable and intensely hot plasma jet, which would not spread out however far it travelled. The beam would be given a known, time-coded modulation as an identity marker.

Aiming and steering such a beam would pose tricky problems. How to sweep it accurately across the face of a planet or asteroid from many millions of kilometres away? Once the beam was locked on a path intersecting that of the target, small variations of its energy might slightly alter its trajectory through the interplanetary field, giving the required scan. At its destination, the beam would induce $X$-ray and visible luminescence at the point of impact, and release backscattered and secondary particles from it. The luminescence would be easier to detect. Since it would be code-modulated at the beam frequency, any crude earthbound telescope trained in its general direction could pick it up. Background illumination from the target, the atmosphere and so on, would be unmodulated and easily rejected. The round-trip delay revealed by the modulation would give information on the fields traversed by the beam. Aimed at an asteroid with no atmosphere, a metre-wide beam could resolve features of its surface on the same scale, and reveal their composition from their X-ray fluorescence. Aimed at a planet, the beam should induce strong atmospheric aurorae, illuminating its meteorology. Aimed at the Moon, it could write luminous advertising slogans visible from Earth, to pay for the project. David Jones 